\title{
Cellular crowding guides and debundles the microtubule cytoskeleton
}

\author{
A. Z. Płochocka,, , * N. A. Bulgakova, ${ }^{2, \text { † }}$ and L. Chumakova ${ }^{3,7}$ \\ ${ }^{1}$ Center for Computational Biology and Center for Computational Mathematics, Flatiron Institute, New York, NY, USA, 10010 \\ ${ }^{2}$ Department of Biomedical Science, The University of Sheffield, Sheffield, UK, S10 2TN \\ ${ }^{3}$ Maxwell Institute for Mathematical Sciences, School of Mathematics, \\ The University of Edinburgh, Edinburgh, UK, EH9 3FD
}

\begin{abstract}
Cytoplasm is densely packed with macromolecules causing cellular crowding, which alters interactions inside cells and differs between biological systems. Here we investigate the impact of crowding on microtubule cytoskeleton organization. Using mathematical modelling, we find that only anisotropic crowding affects the mean microtubule direction, but any crowding reduces the number of microtubules that form bundles. We validate these predictions in vivo using Drosophila follicular epithelium. Since cellular components are transported along microtubules, our results identify cellular crowding as a novel regulator of this transport and cell organization.
\end{abstract}

Subject Areas: Biological Physics, Complex Systems, Interdisciplinary Physics

Distribution of different components inside cells is cru- ${ }_{54}$ cial for cellular, and therefore, organism function. In or- ${ }_{55}$ der for organelles to be delivered to their corresponding ${ }_{56}$ biologically relevant locations inside the cell, they are ${ }_{57}$ transported via vehicles (motor proteins) along tracks ${ }_{58}$ (microtubule cytoskeleton). The microtubules (MTs) ${ }_{59}$ forming these tracks are polarized and highly dynamic 60 filaments [1], as their plus-ends undergo dynamic insta- ${ }_{61}$ bility. In particular, MTs are either growing or shrink- ${ }_{62}$ ing and can switch between the two states. Despite this ${ }_{63}$ highly dynamic behavior of individual MTs, they self- ${ }_{64}$ organize into a network, the dynamics of which reaches ${ }_{65}$ a steady-state. This steady state is often driven by cell- ${ }_{60}$ scale features, e.g. cell geometry and spatial distribution ${ }_{67}$ of MT stable minus-ends [2 4].

The properties of the MT network are crucial for cell 69 function. In particular, the mean MT direction is linked 70 to the large-scale direction of transport and cytoplasmic 71 flows [5-7]. The efficacy of intracellular transport ad- 72 ditionally depends on the MT bundling, which occurs 73 in many experimental systems [8. It is defined as the 74 case when two or more MTs are closely apposed, often 75 connected by cross-linking proteins 9 . The presence of 76 bundling promotes the transport by increasing the prob- 77 ability of a motor protein reattachment to a MT upon 78 fall-off [10, 11.

However, the MT network does not exist in isola- 80 tion, but rather in a crowded cytoplasm densely packed ${ }^{81}$ with biopolymers 12 . This dense packing with macro- 82 molecules can make the cell interior either isotropic 83 or anisotropic [12 15]. The significance of cytoplasmic ${ }^{84}$ crowding is seen in protein folding, where it speeds up 85 transition-limited reactions while slowing down diffusion- 86 limited reactions [13, 16]. Additionally, the crowding cre- 87 ates potential barriers to growing MTs. The only model 88 to date that considers the MTs in the context of crowd- 89 ing analyzes the creation of traffic jams by kinesin-8 [17, 90 whereas the effects of crowding on MTs themselves re- 91 main unknown. In this paper we focus on how cellular 92 crowding and its anisotropy affect MT self-organization.

To address this, we combine stochastic simulations, analytical models and in vivo experiments. We model cellular crowding as barriers in the cytoplasm, where their positions are either statistically isotropic or anisotropic, and homogeneous or discrete. We discover that all barrier types reduce MT bundling, whereas only anisotropic barriers alter their main direction. We validate our predictions in vivo using Drosophila follicular epithelium at late stages of oogenesis [18 20. Altogether, we demonstrate that cellular crowding and its directionality impact on the MT network organization and should be considered when studying MT-related processes in cells.

Model. - As cellular crowding is a universal phenomenon, we turn to a system in which MTs can be modelled without excessive oversimplification. In the epithelial tissue, one of the four major tissue types [3], the cortical MTs are restricted to the thin $1 \mu m$ quasi-2d subapical layer (Fig 1 a , 2]). This allows to model cells as 2d convex domains, in which MTs grow from points on the boundary $\zeta$ into the interior (Fig,1], 21, 22]) at an angle $\theta$ (or $\phi$ ) with respect to the boundary (or the horizontal). All the mathematical model results are presented on elliptical cells, since it is the average cell shape for a given eccentricity [4].

We represent individual MTs as 1d filaments and their dynamic instability via a Markov chain (Fig11, $[2,4,23]$ ), with the of growth $\alpha$, depolymerization $\beta$, rescue $\alpha^{\prime}$ and catastrophe $\beta^{\prime}$ (Fig 1 ;). We set the base rates $\left(\alpha, \beta, \alpha^{\prime}, \beta^{\prime}\right)=(1000,3500,4,1)$ (as in 4]) and change the catastrophe rate $\beta^{\prime}$ depending on the nature of barriers. We assume that crowding does not alter the tubulin concentration in the cytoplasm, and hence $\alpha$ or $\alpha^{\prime}$, whereas the depolymerization rate $\beta$ is independent of it 24]. Upon fully depolymerizing, the MT switches to growing at the rescue rate $\alpha^{\prime}$.

We choose the simplest angle-dependent model of MT interactions (Fig $11 \mathrm{~d},[2]$ ). When a polymerizing MT encounters an existing one at an angle $\theta_{M T}$, it can grow 

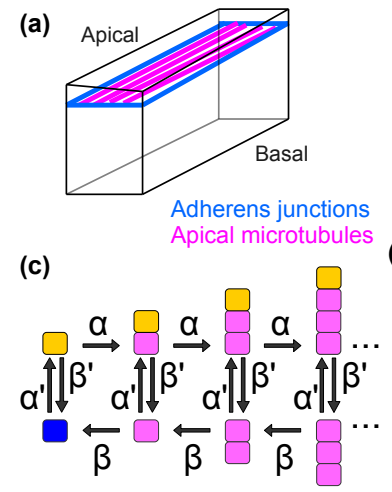

(d)

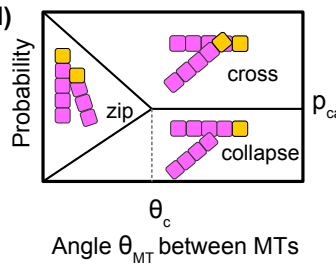

(b)

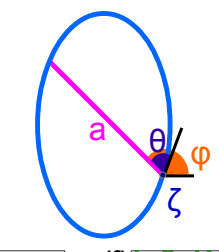

(e)

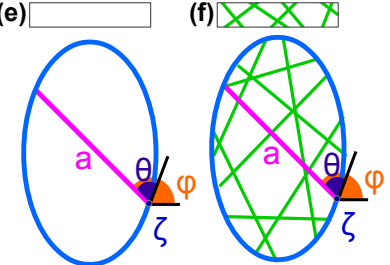

(g)

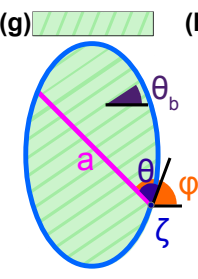

(h)

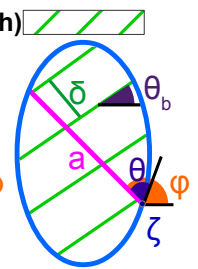

FIG. 1. Model setups of the MT dynamics (a-d) and the ${ }^{120}$ toplasmic crowding (e-h). (a) The apical MTs (magenta) in ${ }^{130}$ epithelial cells are anchored at the adherens junctions $(\text { blue })^{131}$ and grow within the $1 \mu \mathrm{m}$ layer. (b) A MT growing from the ${ }^{132}$ minus-end $\zeta$ on the boundary (blue) into the interior at the ${ }^{133}$ angle $\theta$ (or $\phi$ ) with respect to the boundary (or the horizon-134 tal); $a$ is the cross-section length. (c) Markov chain model ${ }_{135}$ of a MT. The rates of polymerization - $\alpha$, catastrophe - $\beta^{\prime},{ }_{136}$ depolymerization - $\beta$, and rescue (from either the minus-end ${ }_{137}$ (blue) or when depolymerizing (magenta)) - $\alpha^{\prime}$. (d) MT in- ${ }^{137}$ teraction: probabilities of a growing MT to collapse, cross, or ${ }^{138}$ zip parallel to an existing MT as a function of the angle $\theta_{M T}{ }^{139}$ between them. $\theta_{c}$ is the critical angle, $p_{\text {cat }}$ is the probability ${ }^{140}$ of catastrophe. (e-h) The four scenarios of crowding barri-141 ers (green): (e) isotropic homogeneous; (f) isotropic discrete; ${ }_{142}$ (g) anisotropic homogeneous cytoplasm with the angle $\theta_{b}$ of anisotropy; and (h) anisotropic discrete barriers at the angle $\theta_{b}$, with spacing $\delta$. Boxes indicate labels for the crowding models.

parallel to it (zipping), forming a bundle 25. Since $\mathrm{MTs}_{145}$ cannot bend beyond a certain critical angle $\theta_{c}$ due to $\mathrm{to}_{146}$ their rigidity [26], if $\theta_{M T}>\theta_{c}$, the oncoming MT under- ${ }_{147}$ goes catastrophe with probability $p_{\text {cat }}$ and crosses otherwise; and for $\theta_{M T}<\theta_{c}$, it collapses, crosses or zips with ${ }_{149}$ probabilities $\frac{\theta_{\mathrm{MT}}}{\theta_{c}} p_{c a t}, \frac{\theta_{\mathrm{MT}}}{\theta_{c}}\left(1-p_{c a t}\right), 1-\frac{\theta_{\mathrm{MT}}}{\theta_{c}}$ respectively ${ }_{{ }_{150}}{ }^{149}$

To systematically study cellular crowding, we exam-151 ine four barrier placement scenarios named after the ter-152 minology in turbulence. (1) Isotropic homogeneous 153 (Fig,11): the simplified limiting case with small biopoly-154 mers, whose distribution is homogeneous and isotropic,155 is modeled by uniformly increasing the base value of the 150 catastrophe rate $\beta^{\prime}$. (2) Isotropic discrete (Fig 1f):157 when the biopolymers are not aligned, but their distri-158 bution is not homogeneous, e.g. cortical actin mesh [27, 159 they are modelled as discrete random barriers. Upon 160 encountering a barrier, MTs collapse with the probabil-161 ity $p_{b}$, increasing the catastrophe rate from $\beta^{\prime}$ to $\frac{\alpha p_{b}}{1-p_{b}}$. (3) Anisotropic homogeneous (Fig $1 \mathrm{~g}$ ): when the biopolymers are aligned, but in the limiting case of being very close to each other, they are modeled as a barrier field at an angle $\theta_{b}$. Here the catastrophe rate $\tilde{\beta}^{\prime}(\psi)=|\cos \psi| \beta^{\prime}+|\sin \psi| \alpha p_{b} /\left(1-p_{b}\right)$ depends on the angle between the MTs and the barriers $\psi=\phi-\theta_{b}$, increasing from the base rate $\beta^{\prime}$ to the $\frac{\alpha p_{b}}{1-p_{b}}$ when MTs are perpendicular to the barriers. (4) Anisotropic discrete (Fig $1 \mathrm{~h}$ ): The barriers, e.g. actin cables, separated by $\delta$ are placed at the angle $\theta_{b}$ with respect to the horizontal, and the MTs collapse at barriers with the probability $p_{b}$. Since the time-scale of the barrier dynamics in vivo (e.g. actin cables) is much longer than the MT growth cycle (15sec, [2]), we model them as stationary.

Microtubule organization. - For reported parameter ranges of $\beta^{\prime}$ (4 and the references therein), the MT organization is not affected by isotropic crowding (Fig 2 a,b), since homogeneous crowding is the limiting case of infinitely close random barriers, and the MT organization is not sensitive to uniformly changing $\beta^{\prime}$. 4 . Since $\beta^{\prime}$ has not been measured for crowding scenarios, we investigated increased $p_{b}$ corresponding to $\beta^{\prime}$ much higher than the reported range. This progressively weakened the effect of cell geometry 2, 4, reducing MT alignment with the cell major axis (Fig 2 a, $\left.\mathrm{b} \beta^{\prime}=5\right)$.

By contrast, anisotropic crowding introduces competition between the cell geometry and barriers: the former aligns the MTs along the cell major axis, and the latter along the direction of anisotropy (Fig, 2k,d). Since the MT angle distribution does not depend on the interaction parameters $\left(\theta_{c}, p_{\text {cat }}\right)$ (see SI, Fig.S1), we used the analytical distribution

$$
\rho(\phi)=\frac{1}{M} \int \frac{q \int_{0}^{\tilde{a}} y e^{-\int_{0}^{y} p(s) d s} d y}{\frac{1}{\alpha^{\prime}}+\frac{q}{\pi} \int_{0}^{\pi} \int_{0}^{a} e^{-\int_{0}^{y} p(s) d s} d y d \theta} d \zeta,
$$

which assumes non-interacting MTs, to analyze its dependence on the barrier strength (for the derivation and the versions for different crowding scenarios see SI section $\mathrm{C})$. Here $M$ is the normalization constant, $a(\zeta, \theta)=\tilde{a}(\zeta, \phi)$ is the cell cross-section, the parameters $p(\cdot)=\frac{\beta^{\prime}(\cdot)}{\alpha}-\frac{\alpha^{\prime}}{\beta}$ and $q=\frac{1}{\alpha}+\frac{1}{\beta}$, where $\beta^{\prime}(\cdot)$ varies depending on the crowding scenario. For both cases of homogeneous and discrete barriers, we altered the barrier strength $p_{b}$ for non-elongated and elongated cells $(e c c=0.7$ and $e c c=0.98)$, while keeping $\left(\alpha, \beta, \alpha^{\prime}\right)$ and $\left(p_{c a t}, \theta_{c}\right)$, constant $(\mathrm{Fig} 1 \mathrm{~g}, \mathrm{~h})$. For weak barriers, the MT angle distribution is determined by the cell shape, with its peak at the cell major axis angle $\left(90^{\circ}\right)$. With increasing barrier strength, the MTs progressively align with the anisotropy. The rate of this transition depends on the cell geometry and the barrier strength. For elongated cells the effect of the geometry is stronger than for the non-elongated ones, and the MTs align with the cell major axis for larger $p_{b}$. Since the continuous crowding is 
(a) Isotropic Homogeneous
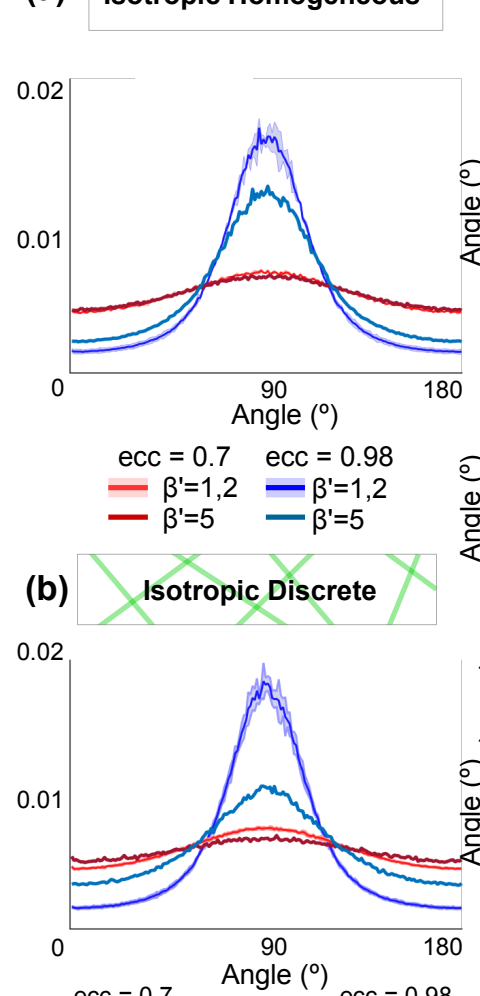

$\mathrm{p}_{\mathrm{b}}=0.001,0.002,0.005$
$\mathrm{~N}_{\mathrm{b}}=20,50$
$\mathrm{P}_{\mathrm{b}}=0.1, \mathrm{~N}_{\mathrm{b}}=0.001,0.002,0.005$
$\mathrm{~N}_{\mathrm{b}}=20,50$

$\mathrm{p}_{\mathrm{b}}=0.1, \mathrm{~N}_{\mathrm{b}}=50 \quad-\mathrm{p}_{\mathrm{b}}=0.1, \mathrm{~N}_{\mathrm{b}}=50$ (c)

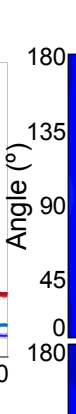

Anisotropic Homogeneous

ecc $=0.7$

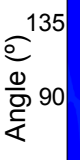

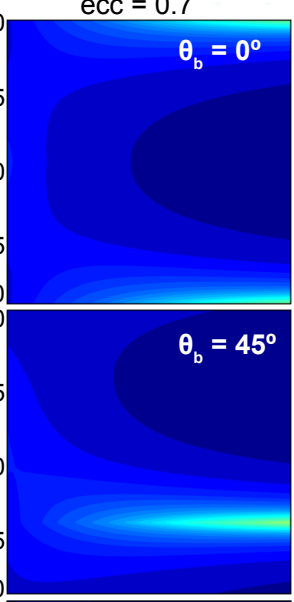

$\theta_{b}=45^{\circ}$

ecc $=0.98$
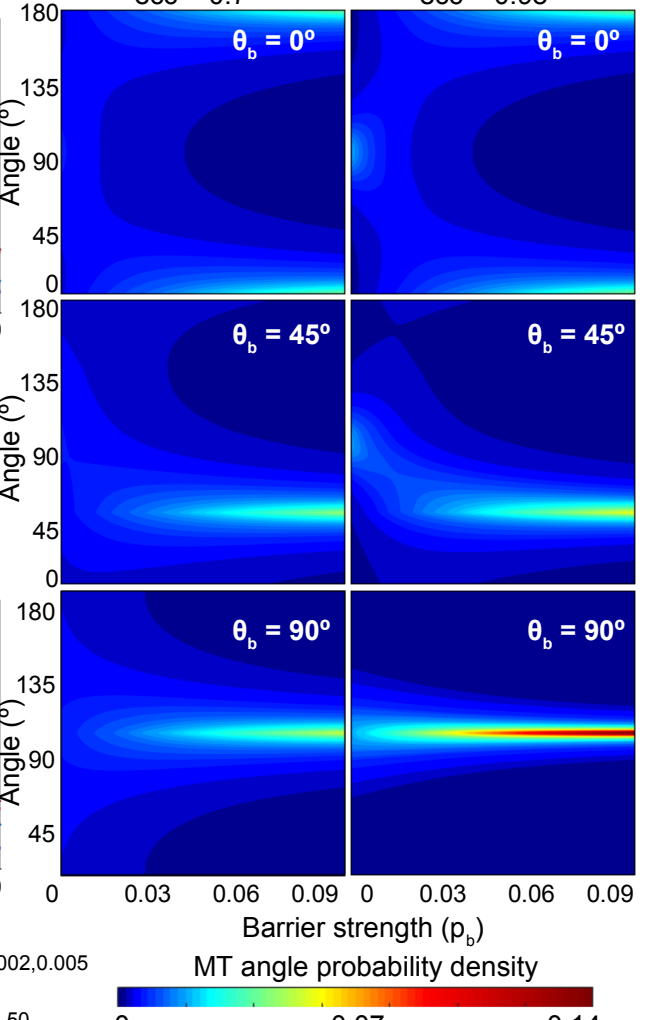

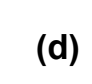

(d)
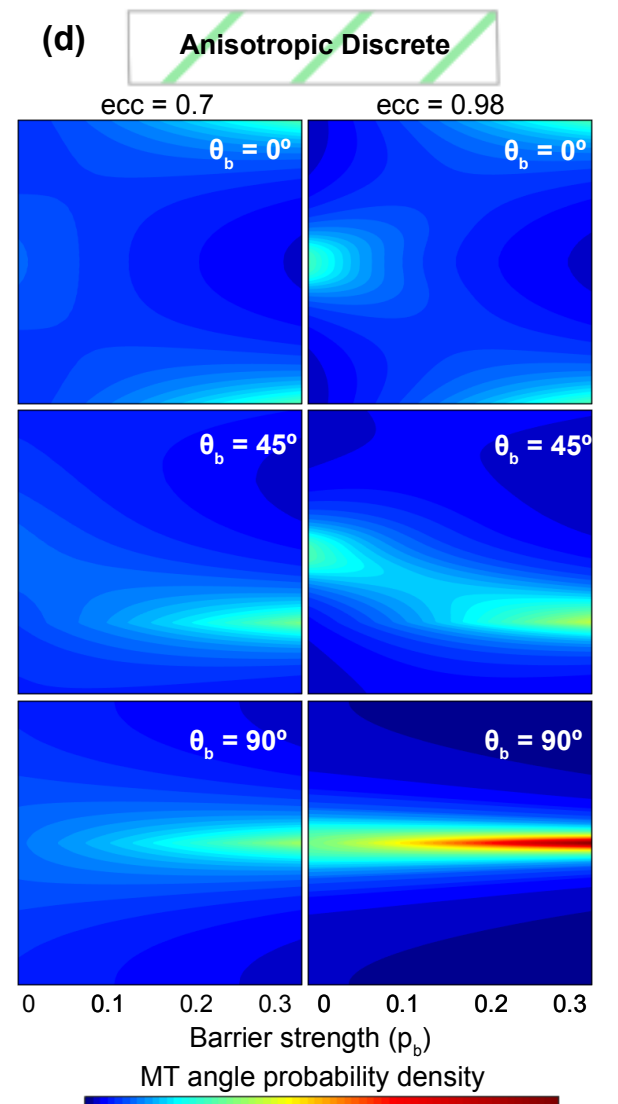

0

0.07

0.14

FIG. 2. Cellular crowding effect on the MT angle distribution in elongated $(e c c=0.98)$ and non-elongated $(e c c=0.7)$ cells. (a-b) The MT angle distributions for isotropic homogeneous (a) and discrete (b) crowding, for ecc = 0.95 (purple), and $e c c=0.7(\mathrm{red})$. Robust distributions for the reported values of $\beta^{\prime}=1,2$, mean (solid) and the standard deviation (envelope). Reduced effect of cell geometry for $\beta^{\prime}=5$ (blue curve). 500 stochastic simulations were run for parameter combination; $p_{b}=0.001,0.002,0.005,0.1$; the number of barriers $N_{b}$ was varied to keep the barrier density approximately constant: $N_{b}=20,50$ for $e c c=0.7$ and $N_{b}=72,179$ for $e c c=0.98$. (c-d) Analytic MT angle distributions for anisotropic homogeneous (c) and discrete (D) crowding as a function of the barrier strengths $p_{b}$ for three barrier angles $\theta_{b}$. In (d) $\delta=10$. The remaining MT instability parameter were kept at their base values.

the limiting case of infinitely close barriers, the MTs align 180 with anisotropy at smaller $p_{b}$, comparing to the discrete ${ }_{181}$ barrier case (see SI Section D for the study of varying $\delta$ ).182

Validation. - We then validated the model pre- ${ }^{183}$ dictions in vivo. As the strongest effect on MT self- ${ }^{184}$ organization is predicted for anisotropic barriers, we used ${ }^{185}$ Drosophila follicular epithelium, where during late oo-186 genesis (Stage 12, SI Section A) the MTs co-exist with ${ }^{187}$ highly aligned densely packed actin cables (Fig 3 a, $\mathrm{b})$. In $^{188}$ the absence of anisotropic crowding, as in the Drosophila $a^{189}$ embryonic epidermis, MTs orient along the main cell axis ${ }^{190}$ 2]. To explore if the actin cables reorient the network, ${ }^{191}$ the cells were rotated to have $0^{\circ}$ major axis angle. As ${ }^{192}$ expected, when not accounted for the actin cable direc-193 tions, the MT network direction was unbiased (Fig 3k).194 After flipping the cell images to have the positive angle195 of actin, the MTs were more likely to have a positive di-196 rection $(\mathrm{p}<0.0001$, Fig $3 \mathrm{p})$. This bias was stronger for ${ }_{197}$ cells with larger differences between the cell major axis and actin direction $(\mathrm{p}=0.001$ and $\mathrm{p}=0.0004$ for differences above $15^{\circ}$ and $25^{\circ}$, Fig 3 ;). We concluded that actin cables reorient the MT network, and this effect increases with the angle difference between the cell major axis and actin cables.

Bundling. - To our surprise, upon removal of actin cables by treating ovaries with Latrunculin A the MT organization changed profoundly (Fig,4a, 28). The MTs appeared more bundled, forming thicker and brighter filaments (Fig, 4 $\mathrm{a}$ ), the average area covered by them was reduced $(p=0.0005$, Fig $4 \mathrm{p})$, while their signal intensity increased ( $p=0.02$, Fig $4 c$ ). We concluded that actin cables inhibit bundling in vivo.

To explore it further via modelling, we introduced the bundling factor as the ratio of MT lengths in bundles to their total length (Fig $4 \mathrm{~d}, \mathrm{e})$. In all crowding models, the bundling factor was reduced in the presence of bar- 
(a)

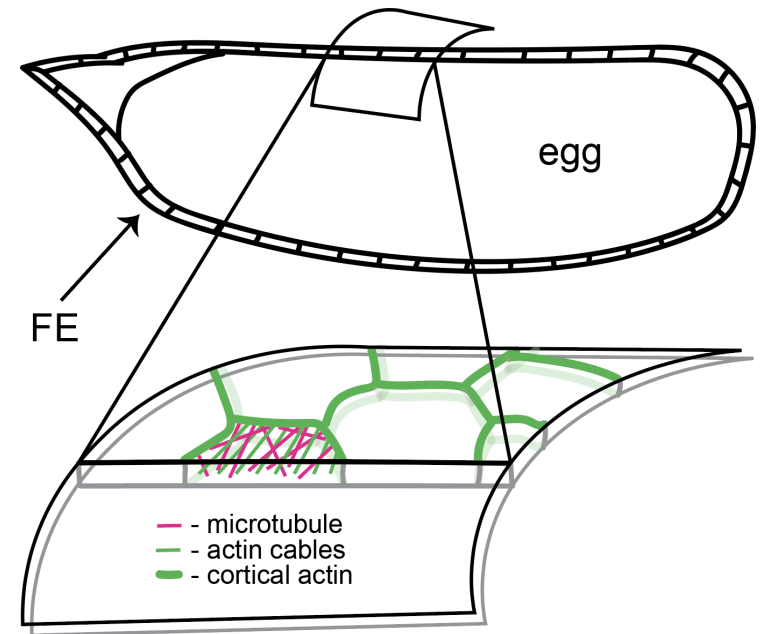

(b)

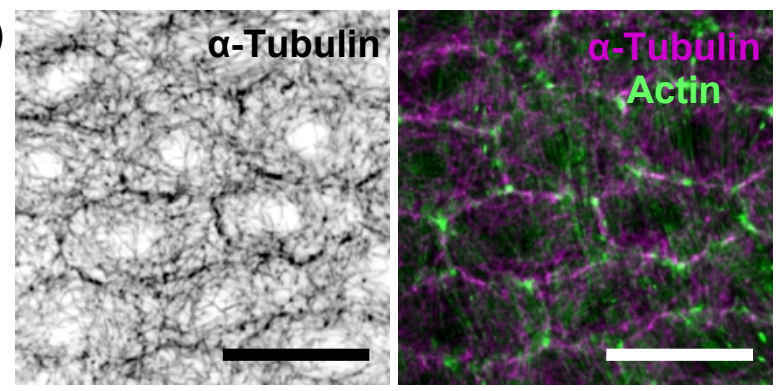

(c)

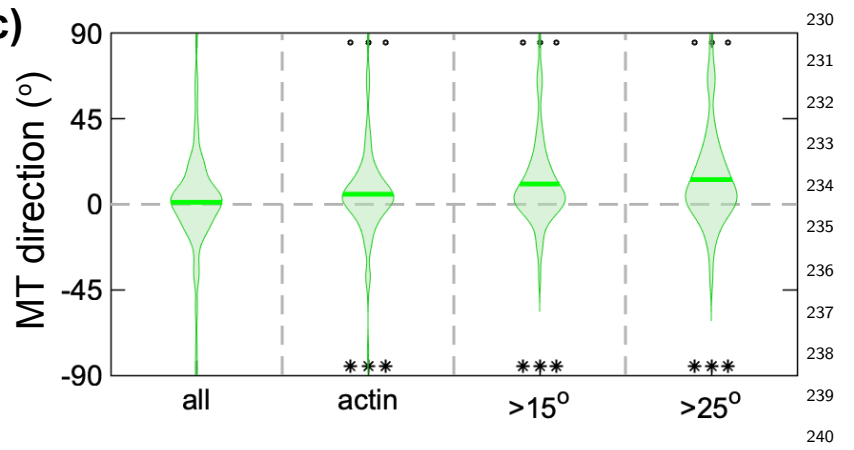

FIG. 3. The effect of actin cables in the Drosophila follicular ${ }^{241}$ epithelium on the MT mean direction. (a) Schematic of the242 follicular epithelium (FE): a layer of thin cells surrounding ${ }_{243}$ the egg chamber with a closer view of $\mathrm{FE}$ (bottom): $\mathrm{MTs}_{244}$ (magenta) and actin (green). (b) Example of follicular cells stained for MTs (grey, left; magenta, right) and actin (green, right). The scale bar is $10 \mu \mathrm{m}$. (c) The main direction of MT network without normalization to the direction of actin (all), and with normalization: in all cells (actin), and in cells with the angle between their direction and actin greater than $15^{o^{245}}$ $\left(>15^{\circ}\right)$ and $25^{\circ}\left(>25^{\circ}\right)$. ${ }^{* * *}$-p $<0.0001$ to differ from zero; ${ }^{\text {ooo }}{ }_{-}{ }_{247} 46$ $\mathrm{p}<0.001$ in comparison to the non-normalized distributions. ${ }^{247}$

\section{${ }^{20}$}

222 223 224

.

measure: MT bundling, by counting MTs which zip along
each other. Finally, we validated the model of discrete
anisotropic barriers in vivo on the Drosophila follicular
epithelium.
We found that only anisotropic crowding affects the
direction of MT network. This is due to the competition
between the cell geometry aligning it along the cell major
axis 2 , 4 and anisotropic crowding redirecting it along
itself, where the geometry effect is stronger for more elon-
gated cells. The orientation of the MT network directs
intracellular transport $[5]$, which in some biological sys-
tems is required to be other than the cell major axis. For
example, in the follicular epithelium the transmembrane
protein Fat2 accumulates along the boundaries parallel
to the cell major axis 29 . This localization depends on
MTs $[19$, 29, suggesting the need for their reorientation
for the efficient delivery of Fat2 to produce a viable egg.
Therefore, cellular crowding anisotropy provides a pow-
erful tool for a cell to redirect the transport and perform
its correct function.
We showed both in vivo and in silico that cellular
crowding reduces bundling. How this alters efficacy of
intracellular trafficking by molecular motors remains an
open question, as bundling can both increase and de-
crease trafficking by, first, reducing the overall MT den-
sity in the cytoplasm, while increasing the probability of
motor re-attachment after a fall-of a MT, thus facilitat-
ing the cargo reaching the cell boundary. In summary,
cellular crowding, though often overlooked, is an impor-
tant contributor to MT self-organization, and thus to the
correct cellular organization and function.
This research was supported by The Maxwell Insti-
tute Graduate School in Analysis and its Applications,
the Centre for Doctoral Training funded by the UK EP-
SRC grant EP/L016508/01, the Scottish Funding Coun-
cil, Heriot-Watt University and the University of Edin-
burgh (A.Z.P.); BBSRC BB/P007503/1 (N.A.B.); Royal
Society of Edinburgh and the Scottish Government per-
sonal fellowship (L.C.); and the Leverhulme trust grant
RPG-2017-249 (L.C. and N.A.B).

ts reserved. No reuse allowed without permission.
measure: MT bundling, by counting MTs which zip along
each other. Finally, we validated the model of discrete
anisotropic barriers in vivo on the Drosophila follicular
epithelium.
We found that only anisotropic crowding affects the
direction of MT network. This is due to the competition
between the cell geometry aligning it along the cell major
axis [2, 4 and anisotropic crowding redirecting it along
itself, where the geometry effect is stronger for more elon-
gated cells. The orientation of the MT network directs
intracellular transport [5], which in some biological sys-
tems is required to be other than the cell major axis. For
example, in the follicular epithelium the transmembrane
protein Fat2 accumulates along the boundaries parallel
to the cell major axis 29 . This localization depends on
MTs $[19$, 29, suggesting the need for their reorientation
for the efficient delivery of Fat2 to produce a viable egg.
Therefore, cellular crowding anisotropy provides a pow-
erful tool for a cell to redirect the transport and perform
its correct function.
We showed both in vivo and in silico that cellular
crowding reduces bundling. How this alters efficacy of
intracellular trafficking by molecular motors remains an
open question, as bundling can both increase and de-
crease trafficking by, first, reducing the overall MT den-
sity in the cytoplasm, while increasing the probability of
motor re-attachment after a fall-of a MT, thus facilitat-
ing the cargo reaching the cell boundary. In summary,
cellular crowding, though often overlooked, is an impor-
tant contributor to MT self-organization, and thus to the
correct cellular organization and function.
This research was supported by The Maxwell Insti-
tute Graduate School in Analysis and its Applications,
the Centre for Doctoral Training funded by the UK EP-
SRC grant EP/L016508/01, the Scottish Funding Coun-
cil, Heriot-Watt University and the University of Edin-
burgh (A.Z.P.); BBSRC BB/P007503/1 (N.A.B.); Royal
Society of Edinburgh and the Scottish Government per-
sonal fellowship (L.C.); and the Leverhulme trust grant
RPG-2017-249 (L.C. and N.A.B).
a

measure: MT bundling, by counting MTs which zip along
each other. Finally, we validated the model of discrete
anisotropic barriers in vivo on the Drosophila follicular
epithelium.
We found that only anisotropic crowding affects the
direction of MT network. This is due to the competition
between the cell geometry aligning it along the cell major
axis 2 , 4] and anisotropic crowding redirecting it along
itself, where the geometry effect is stronger for more elon-
gated cells. The orientation of the MT network directs
intracellular transport [5], which in some biological sys-
tems is required to be other than the cell major axis. For
example, in the follicular epithelium the transmembrane
protein Fat2 accumulates along the boundaries parallel
to the cell major axis 29 . This localization depends on
MTs 19 , 29, suggesting the need for their reorientation
for the efficient delivery of Fat2 to produce a viable egg.
Therefore, cellular crowding anisotropy provides a pow-
erful tool for a cell to redirect the transport and perform
its correct function.
We showed both in vivo and in silico that cellular
crowding reduces bundling. How this alters efficacy of
intracellular trafficking by molecular motors remains an
open question, as bundling can both increase and de-
crease trafficking by, first, reducing the overall MT den-
sity in the cytoplasm, while increasing the probability of
motor re-attachment after a fall-of a MT, thus facilitat-
ing the cargo reaching the cell boundary. In summary,
cellular crowding, though often overlooked, is an impor-
tant contributor to MT self-organization, and thus to the
correct cellular organization and function.
This research was supported by The Maxwell Insti-
tute Graduate School in Analysis and its Applications,
the Centre for Doctoral Training funded by the UK EP-
SRC grant EP/L016508/01, the Scottish Funding Coun-
cil, Heriot-Watt University and the University of Edin-
burgh (A.Z.P.); BBSRC BB/P007503/1 (N.A.B.); Royal
Society of Edinburgh and the Scottish Government per-
sonal fellowship (L.C.); and the Leverhulme trust grant
RPG-2017-249 (L.C. and N.A.B).
aplochockaflironinstitute org

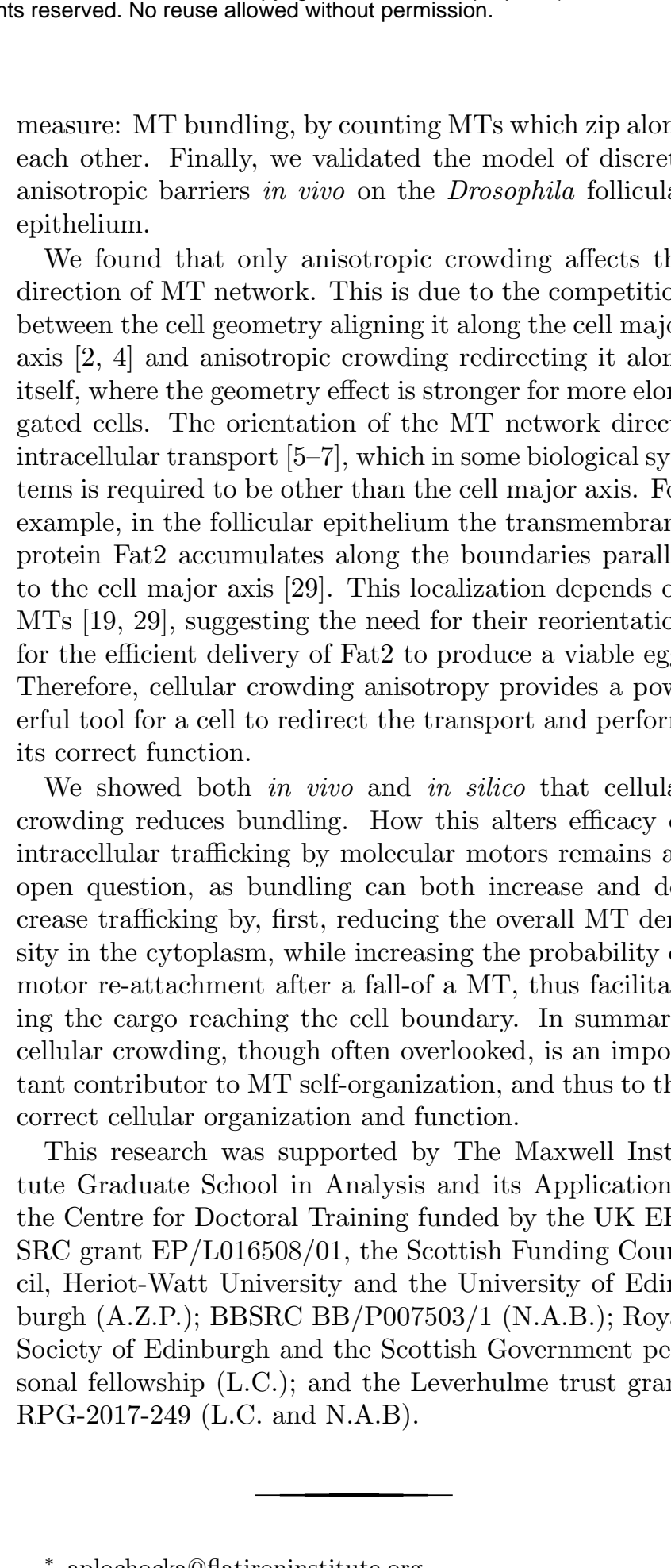

reserved. No reuse allowed without permission.
measure: MT bundling, by counting MTs which zip along
ach other. Finally, we validated the model of discrete
nisotropic barriers in vivo on the Drosophila follicular
pithelium.
We found that only anisotropic crowding affects the
irection of MT network. This is due to the competition
etween the cell geometry aligning it along the cell major
xis [2, 4] and anisotropic crowding redirecting it along
self, where the geometry effect is stronger for more elon-
ated cells. The orientation of the MT network directs
intracellular transport [5], which in some biological sys-
ems is required to be other than the cell major axis. For
xample, in the follicular epithelium the transmembrane
rotein Fat2 accumulates along the boundaries parallel
the cell major axis 29 . This localization depends on
ITs 19 , 29, suggesting the need for their reorientation
the efficient delivery of Fat2 to produce a viable egg.
Therefore, cellular crowding anisotropy provides a pow-
erful tool for a cell to redirect the transport and perform
*

measure: MT bundling, by counting MTs which zip along
each other. Finally, we validated the model of discrete
anisotropic barriers in vivo on the Drosophila follicular
epithelium.
We found that only anisotropic crowding affects the
direction of MT network. This is due to the competition
between the cell geometry aligning it along the cell major
axis 2 , 4 and anisotropic crowding redirecting it along
itself, where the geometry effect is stronger for more elon-
gated cells. The orientation of the MT network directs
intracellular transport [5], which in some biological sys-
tems is required to be other than the cell major axis. For
example, in the follicular epithelium the transmembrane
protein Fat2 accumulates along the boundaries parallel
to the cell major axis 29 . This localization depends on
MTs $[19$, 29 , suggesting the need for their reorientation
for the efficient delivery of Fat2 to produce a viable egg.
Therefore, cellular crowding anisotropy provides a pow-
erful tool for a cell to redirect the transport and perform
its correct function.
We showed both in vivo and in silico that cellular
crowding reduces bundling. How this alters efficacy of
intracellular trafficking by molecular motors remains an
open question, as bundling can both increase and de-
crease trafficking by, first, reducing the overall MT den-
sity in the cytoplasm, while increasing the probability of
motor re-attachment after a fall-of a MT, thus facilitat-
ing the cargo reaching the cell boundary. In summary,
cellular crowding, though often overlooked, is an impor-
tant contributor to MT self-organization, and thus to the
correct cellular organization and function.
This research was supported by The Maxwell Insti-
tute Graduate School in Analysis and its Applications,
the Centre for Doctoral Training funded by the UK EP-
SRC grant EP/L016508/01, the Scottish Funding Coun-
cil, Heriot-Watt University and the University of Edin-
burgh (A.Z.P.); BBSRC BB/P007503/1 (N.A.B.); Royal
Society of Edinburgh and the Scottish Government per-
sonal fellowship (L.C.); and the Leverhulme trust grant
RPG-2017-249 (L.C. and N.A.B).
a

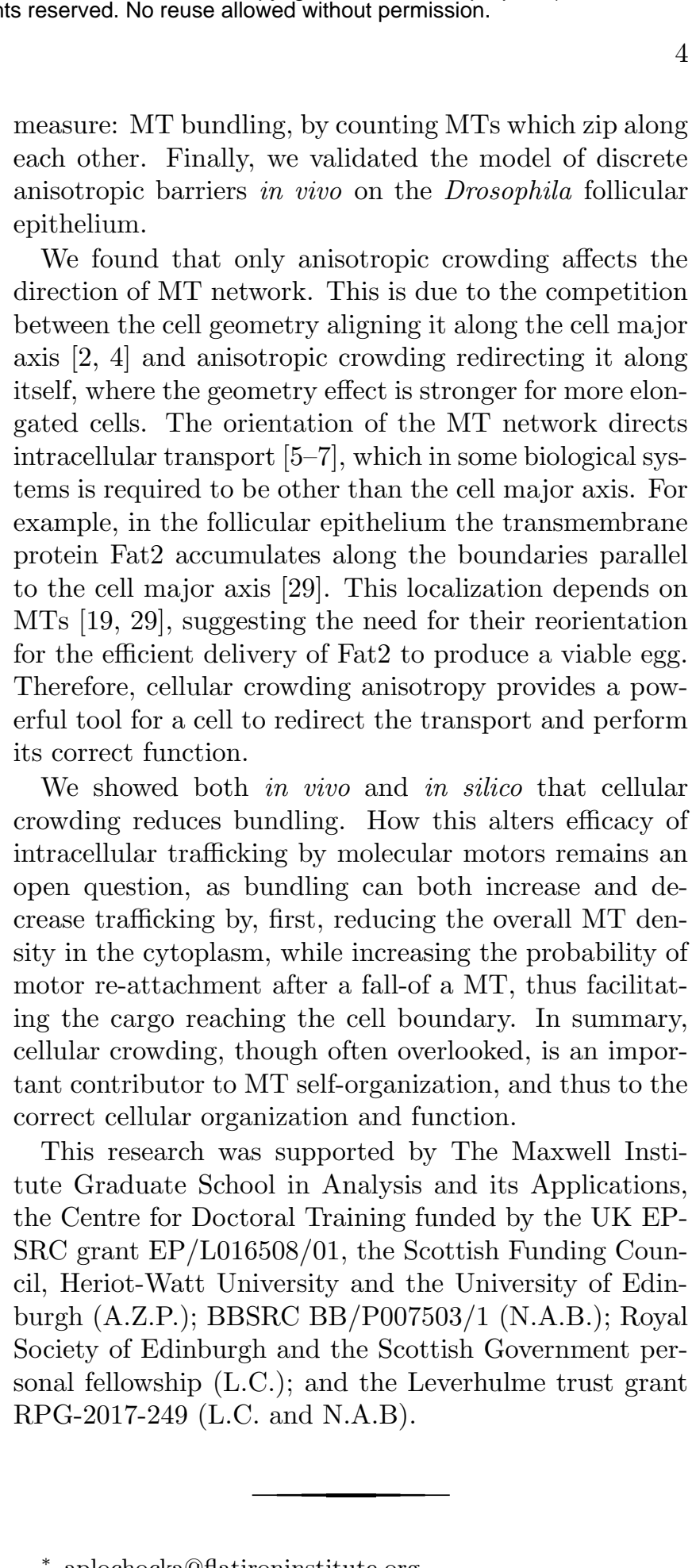

measure: MT bundling, by counting MTs which zip along
each other. Finally, we validated the model of discrete
anisotropic barriers in vivo on the Drosophila follicular
epithelium.
We found that only anisotropic crowding affects the
direction of MT network. This is due to the competition
between the cell geometry aligning it along the cell major
axis 2 , 4 and anisotropic crowding redirecting it along
itself, where the geometry effect is stronger for more elon-
gated cells. The orientation of the MT network directs
intracellular transport [5], which in some biological sys-
tems is required to be other than the cell major axis. For
example, in the follicular epithelium the transmembrane
protein Fat2 accumulates along the boundaries parallel
to the cell major axis 29 . This localization depends on
MTs $[19$, 29 , suggesting the need for their reorientation
for the efficient delivery of Fat2 to produce a viable egg.
Therefore, cellular crowding anisotropy provides a pow-
erful tool for a cell to redirect the transport and perform
its correct function.
We showed both in vivo and in silico that cellular
crowding reduces bundling. How this alters efficacy of
intracellular trafficking by molecular motors remains an
open question, as bundling can both increase and de-
crease trafficking by, first, reducing the overall MT den-
sity in the cytoplasm, while increasing the probability of
motor re-attachment after a fall-of a MT, thus facilitat-
ing the cargo reaching the cell boundary. In summary,
cellular crowding, though often overlooked, is an impor-
tant contributor to MT self-organization, and thus to the
correct cellular organization and function.
This research was supported by The Maxwell Insti-
tute Graduate School in Analysis and its Applications,
the Centre for Doctoral Training funded by the UK EP-
SRC grant EP/L016508/01, the Scottish Funding Coun-
cil, Heriot-Watt University and the University of Edin-
burgh (A.Z.P.); BBSRC BB/P007503/1 (N.A.B.); Royal
Society of Edinburgh and the Scottish Government per-
sonal fellowship (L.C.); and the Leverhulme trust grant
RPG-2017-249 (L.C. and N.A.B).
a

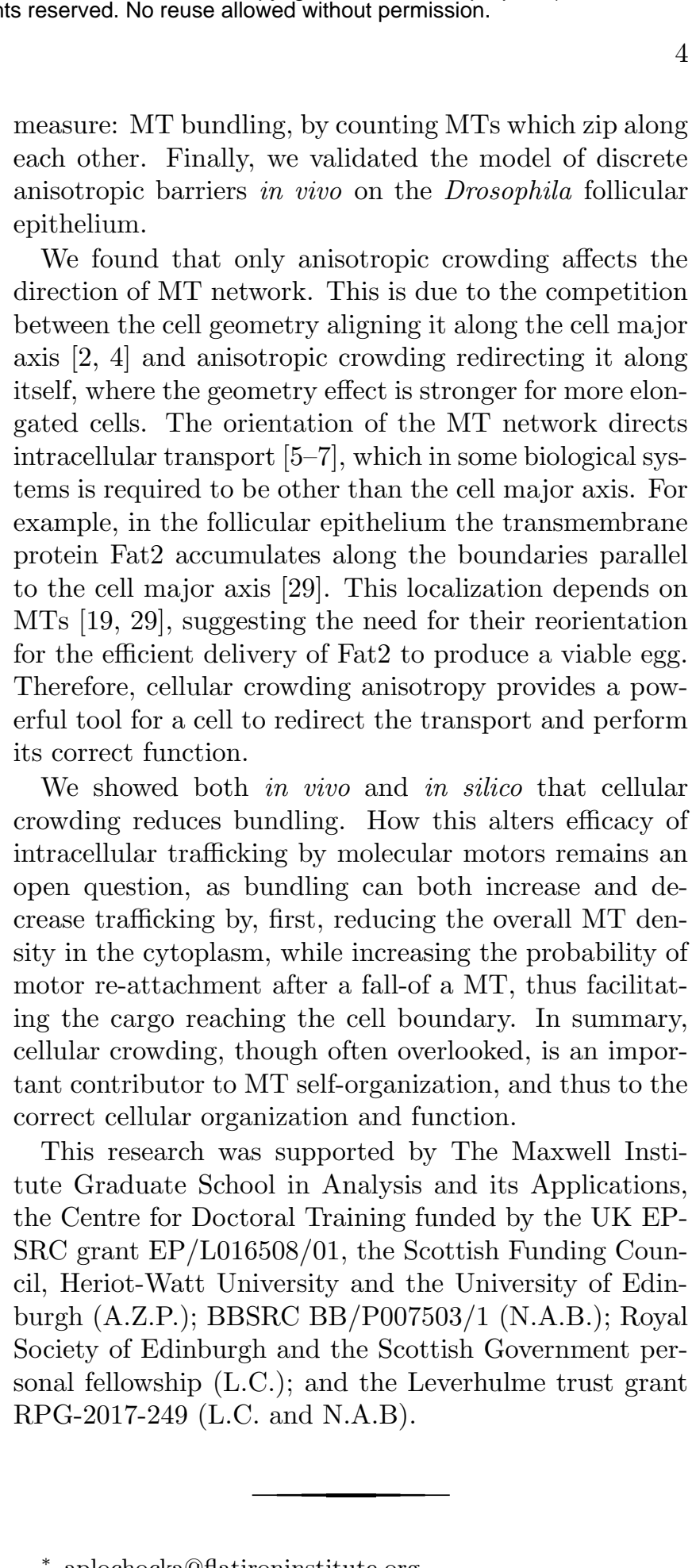

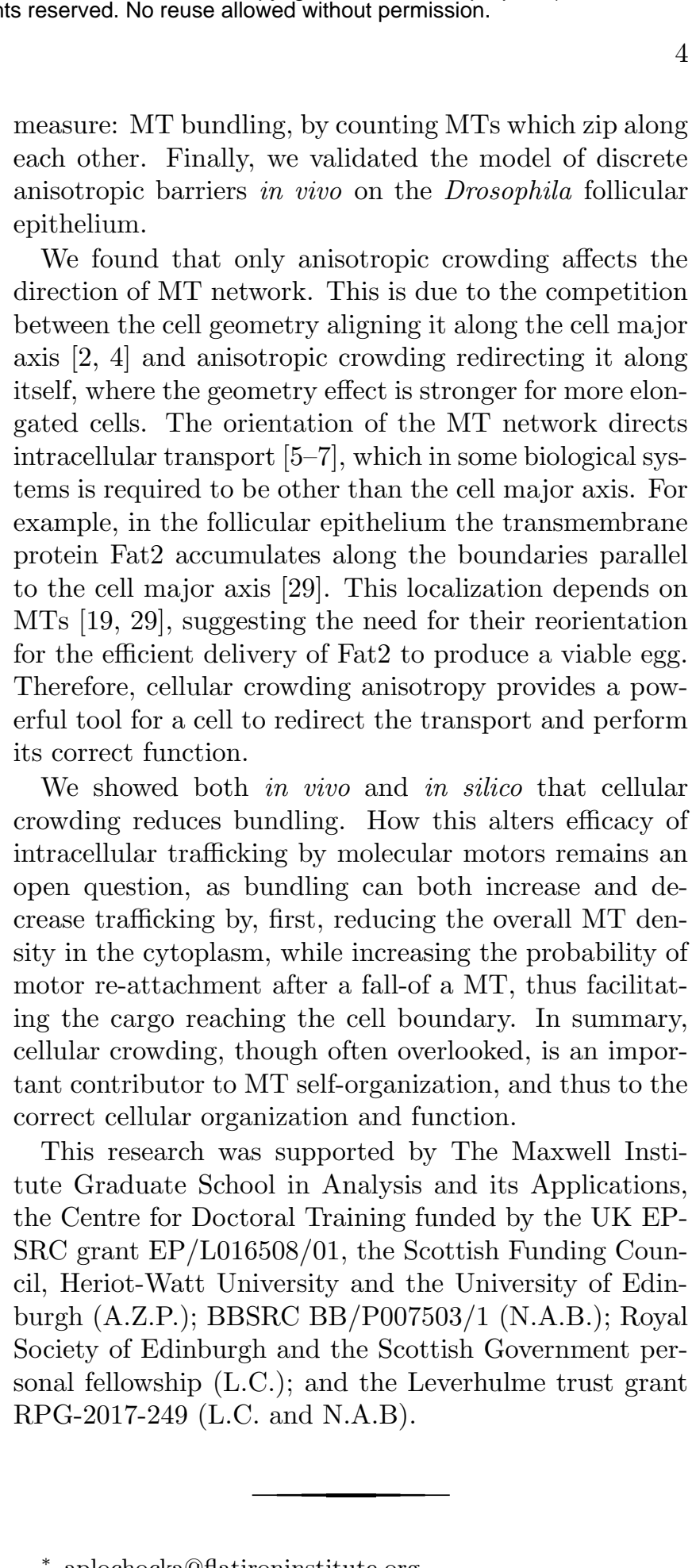

measure: MT bundling, by counting MTs which zip along
each other. Finally, we validated the model of discrete
anisotropic barriers in vivo on the Drosophila follicular
epithelium.
We found that only anisotropic crowding affects the
direction of MT network. This is due to the competition
between the cell geometry aligning it along the cell major
axis 2 , 4 and anisotropic crowding redirecting it along
itself, where the geometry effect is stronger for more elon-
gated cells. The orientation of the MT network directs
intracellular transport [5], which in some biological sys-
tems is required to be other than the cell major axis. For
example, in the follicular epithelium the transmembrane
protein Fat2 accumulates along the boundaries parallel
to the cell major axis 29 . This localization depends on
MTs $[19$, 29 , suggesting the need for their reorientation
for the efficient delivery of Fat2 to produce a viable egg.
Therefore, cellular crowding anisotropy provides a pow-
erful tool for a cell to redirect the transport and perform
its correct function.
We showed both in vivo and in silico that cellular
crowding reduces bundling. How this alters efficacy of
intracellular trafficking by molecular motors remains an
open question, as bundling can both increase and de-
crease trafficking by, first, reducing the overall MT den-
sity in the cytoplasm, while increasing the probability of
motor re-attachment after a fall-of a MT, thus facilitat-
ing the cargo reaching the cell boundary. In summary,
cellular crowding, though often overlooked, is an impor-
tant contributor to MT self-organization, and thus to the
correct cellular organization and function.
This research was supported by The Maxwell Insti-
tute Graduate School in Analysis and its Applications,
the Centre for Doctoral Training funded by the UK EP-
SRC grant EP/L016508/01, the Scottish Funding Coun-
cil, Heriot-Watt University and the University of Edin-
burgh (A.Z.P.); BBSRC BB/P007503/1 (N.A.B.); Royal
Society of Edinburgh and the Scottish Government per-
sonal fellowship (L.C.); and the Leverhulme trust grant
RPG-2017-249 (L.C. and N.A.B).
a

measure: MT bundling, by counting MTs which zip along
each other. Finally, we validated the model of discrete
anisotropic barriers in vivo on the Drosophila follicular
epithelium.
We found that only anisotropic crowding affects the
direction of MT network. This is due to the competition
between the cell geometry aligning it along the cell major
axis 2 , 4 and anisotropic crowding redirecting it along
itself, where the geometry effect is stronger for more elon-
gated cells. The orientation of the MT network directs
intracellular transport [5], which in some biological sys-
tems is required to be other than the cell major axis. For
example, in the follicular epithelium the transmembrane
protein Fat2 accumulates along the boundaries parallel
to the cell major axis 29 . This localization depends on
MTs $[19$, 29 , suggesting the need for their reorientation
for the efficient delivery of Fat2 to produce a viable egg.
Therefore, cellular crowding anisotropy provides a pow-
erful tool for a cell to redirect the transport and perform
its correct function.
We showed both in vivo and in silico that cellular
crowding reduces bundling. How this alters efficacy of
intracellular trafficking by molecular motors remains an
open question, as bundling can both increase and de-
crease trafficking by, first, reducing the overall MT den-
sity in the cytoplasm, while increasing the probability of
motor re-attachment after a fall-of a MT, thus facilitat-
ing the cargo reaching the cell boundary. In summary,
cellular crowding, though often overlooked, is an impor-
tant contributor to MT self-organization, and thus to the
correct cellular organization and function.
This research was supported by The Maxwell Insti-
tute Graduate School in Analysis and its Applications,
the Centre for Doctoral Training funded by the UK EP-
SRC grant EP/L016508/01, the Scottish Funding Coun-
cil, Heriot-Watt University and the University of Edin-
burgh (A.Z.P.); BBSRC BB/P007503/1 (N.A.B.); Royal
Society of Edinburgh and the Scottish Government per-
sonal fellowship (L.C.); and the Leverhulme trust grant
RPG-2017-249 (L.C. and N.A.B).
a

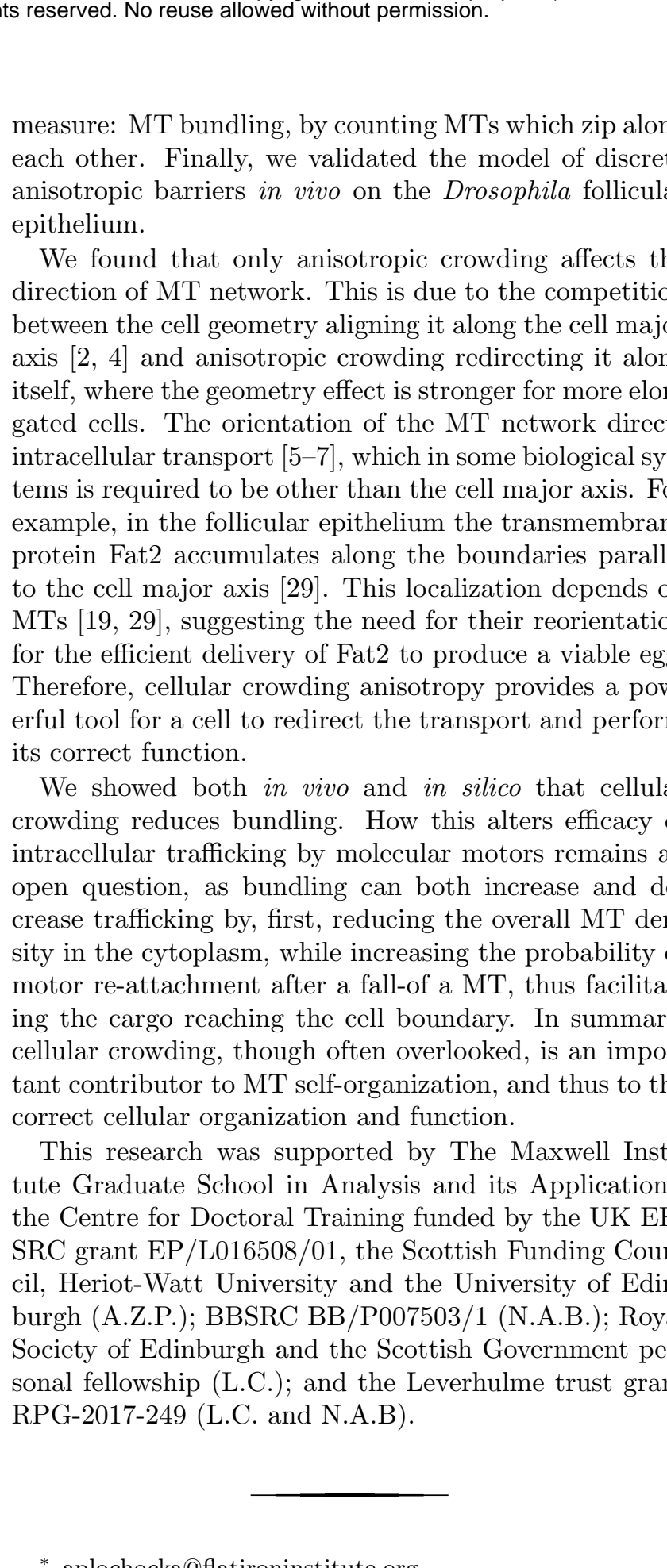

* aplochocka@flatironinstitute.org

† n.bulgakova@sheffield.ac.uk

¥ lyuba.chumakova@ed.ac.uk

[1] G. J. Brouhard, Molecular biology of the cell 26, 1207 (2015).

[2] J. M. Gomez, L. Chumakova, N. A. Bulgakova, and N. H. Brown, Nature Communications 7, 13172 (2016).

[3] A. Muroyama and T. Lechler, Development 144, 3012 (2017).

[4] A. Z. Płochocka, A. M. Davie, N. A. Bulgakova, and L. Chumakova, bioRxiv, 825786 (2019).

[5] K. Barlan and V. I. Gelfand, Cold Spring Harbor perspectives in biology 9, a025817 (2017).

[6] S. Ganguly, L. S. Williams, I. M. Palacios, and R. E. 


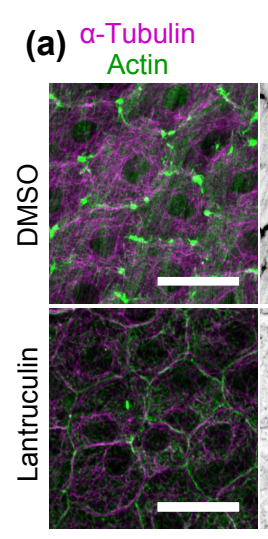

(b)

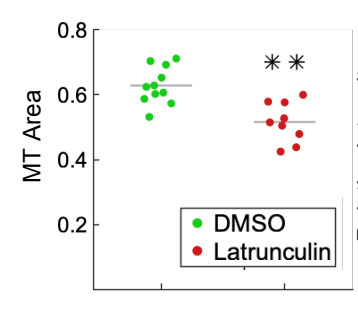

Actin

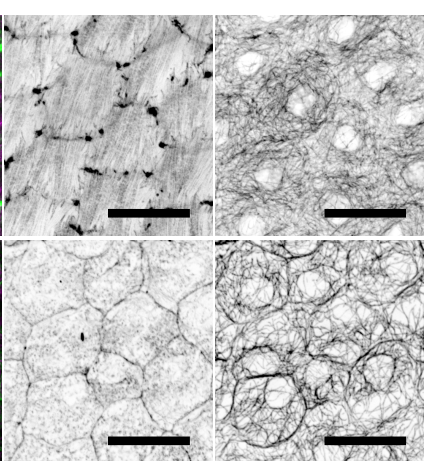

(c)

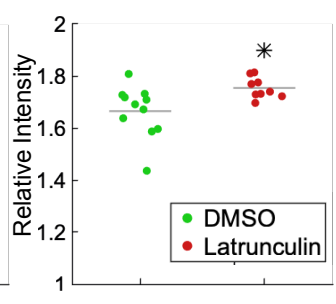

(d)

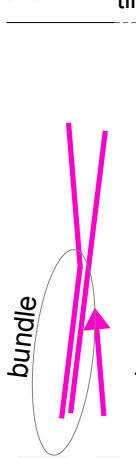

(e)

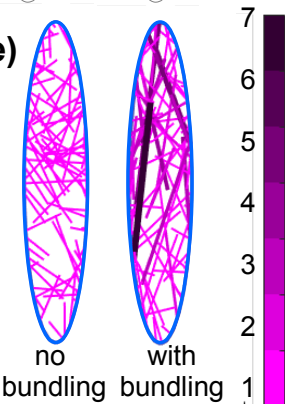

(f)

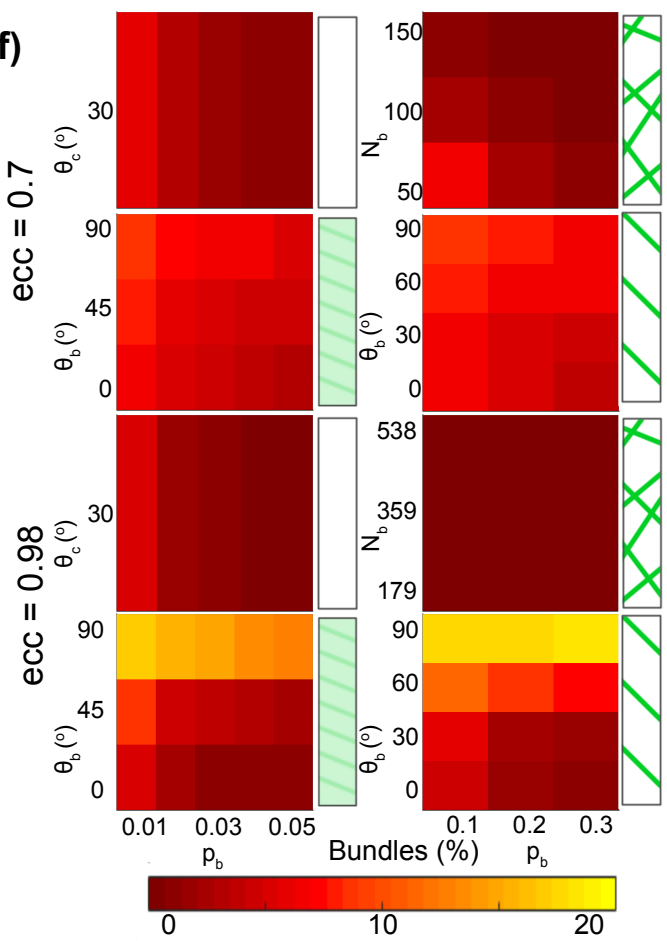

FIG. 4. Effect of cellular crowding on MT bundling. (a) Drosophila follicular epithelium cells in control (top) and with disassembled actin cables after treatment with Latrunculin A (bottom), stained for MTs (magenta - left, grey - right) and actin (green - left, grey - right). The scale bar is $10 \mu \mathrm{m}$. (b) Average area covered by MTs (MT signal area divided by the cell area), and (c) signal relative intensity indicating MT bundling. Each dot represents an individual egg chamber in (b) and (c). ${ }^{*}-\mathrm{p}<0.1$, and ${ }^{* *}$-p=0.0005. (d) Bundle formation. (e) Snapshot of stochastic simulations (ecc =0.98, 200 MTs, $\left(\alpha, \beta, \alpha^{\prime}, \beta^{\prime}\right)=(1000,3500,4,1)$ with non-bundling (left) and bundling MTs $\left(\right.$ right,$\left.\left(\theta_{c}, p_{\text {cat }}\right)=\left(30^{\circ}, 0.01\right)\right)$. (f) Bundling factor $(e c c=0.7$ - top, ecc $=0.98$ - bottom), for the four crowding scenarios (clockwise: isotropic homogeneous, isotropic discrete, anisotropic discrete (with $\delta=10$ ), anisotropic homogeneous) as a function of the barrier strength $p_{b}$ (horizontal axis) and either the number of barriers $N_{b}$ for the isotropic discrete case, or the angle barrier $\theta_{b}$ for the anisotropic cases (vertical axis).

Goldstein, Proceedings of the National Academy of Sci-284 ences 109, 15109 (2012).

[7] S. Roth and J. A. Lynch, Cold Spring Harbor Perspec- ${ }_{286}$ tives in Biology 1, a001891 (2009).

[8] R. J. Hawkins, B. M. Mulder, and S. H. Tindemans, ${ }_{288}$ Physical Review Letters 104, 058103 (2010).

[9] C. E. Walczak and S. L. Shaw, Cell 142, 364 (2010).

[10] L. Balabanian, C. L. Berger, and A. G. Hendricks, Bio- 291 physical journal 113, 1551 (2017).

[11] L. Conway, M. W. Gramlich, S. M. A. Tabei, and J. L.293 Ross, Cytoskeleton 71, 595 (2014).

[12] J. R. Ellis, Trends in Biochemical Sciences 26, $597_{295}$ (2001).

[13] H. Zhou, G. Rivas, and A. P. Minton, Annual Review of 296 Biophysics 37, 375 (2008).

[14] K. Luby-Phelps, in International Review of Cytology (El- ${ }^{298}$ sevier, Amsterdam, Netherlands, 2000), pp. 189-221. 300

[15] Y. Wang, M. Sarkar, A. E. Smith, A. S. Krois, and G. J.301 Pielak, Journal of the American Chemical Society 134, 302 16614 (2012).

[16] J. A. Dix and A. S. Verkman, Annual Review of Bio- ${ }_{304}$ physics 37, 247 (2008).

[17] C. Leduc, K. Padberg-Gehle, V. Varga, D. Helbing,, 306 S. Diez, and J. Howard, Proceedings of the National ${ }_{307}$ Academy of Sciences 109, 6100 (2012).
[18] I. Delon and N. H. Brown, Journal of Cell Science 122, 4363 (2009).

[19] I. Viktorinová and C. Dahmann, Current Biology 23, 1472 (2013).

[20] K. Barlan, M. Cetera, and S. Horne-Badovinac, Developmental Cell 40, 467 (2017).

[21] W. Meng, Y. Mushika, T. Ichii, and M. Takeichi, Cell 135, 948 (2008).

[22] S. S. Goodwin and R. D. Vale, Cell 143, 263 (2010).

[23] C. Peskin, Documenta Mathematica, Extra Volume ICM 3, 633 (1998).

[24] R. A. Walker, E. T. O'Brien, N. K. Pryer, M. F. Soboeiro, W. A. Voter, H. P. Erickson, and E. D. Salmon, The Journal of Cell Biology 107, 1437 (1988).

[25] S. V. Bratman and F. Chang, Trends in Cell Biology 18, 580 (2008).

[26] M. E. Janson, M. E. de Dood, and M. Dogterom, The Journal of Cell Biology 161, 1029 (2003).

[27] F. Eghiaian, A. Rigato, and S. Scheuring, Biophysical Journal 108, 1330 (2015).

[28] M. Coué, S. L. Brenner, and I. S. E. D. Korn, FEBS letters 213, 316 (1987).

[29] I. Viktorinová, T. König, K. Schlichting, and C. Dahmann, Development 136, 4123 (2009). 